\title{
Pierre Michon et l'hétéronomie de la littérature contemporaine
}

\author{
Michel Biron \\ Université McGill
}

S'il est vrai que les écrivains français contemporains sont peu enclins à se réclamer de programmes esthétiques comme au temps des grands mouvements littéraires qui ont marqué la modernité, il reste qu'ils acceptent volontiers de décrire les conditions dans lesquelles ils exercent leur «métier» d'écrivain. Dans une récente enquête intitulée La Condition littéraire. La double vie des écrivains, le sociologue Bernard Lahire a interrogé plus de huit cents écrivains français de la région Rhône-Alpes. Cinq cents d'entre eux ont rempli son questionnaire : c'est donc plus de la moitié des écrivains visés qui ont répondu à l'appel et accepté de commenter leur 
pratique. On voit bien, par ce seul exemple, que les écrivains français contemporains ne se font pas prier pour parler de leur activité littéraire, que ce soit dans les médias ou, comme ici, dans le cadre d'une enquête à visée scientifique. Les écrivains ont beaucoup de choses à dire lorsqu'il est question de leur pratique littéraire, comme on le verra plus loin à partir de l'exemple de Pierre Michon. On pourrait même soutenir que les écrivains d'aujourd'hui, lorsqu'ils parlent de littérature, parlent d'abord et avant tout de leur pratique et non pas d'autre chose. La pratique littéraire suppose une série d'activités bien concrètes, par opposition à l'idée de «règles" ou de «principes». Les écrivains décrivent apparemment plus volontiers des «pratiques de l'art» qu'ils n'énoncent des « règles de l'art», selon le titre de l'ouvrage de Pierre Bourdieu auquel Bernard Lahire répond directement.

Bourdieu, on le sait, a conçu sa sociologie de la littérature en partant du cas de Flaubert, symbole d'une vocation littéraire dont on chercherait en vain l'équivalent dans le monde contemporain. Comme Bourdieu, nous avons coutume de penser les conditions de la littérature moderne à partir du siècle de Flaubert et d'écrivains-théoriciens qui auraient sans doute bien ri s'ils avaient reçu le questionnaire de Bernard Lahire. Mais à bien y penser, il est assez étrange qu'on se représente encore aujourd'hui la littérature à partir d'une figure aussi peu typique que celle de Flaubert, et c'est d'ailleurs l'intérêt de la réflexion menée par Bernard Lahire, qui procède à l'inverse d'un Bourdieu : au lieu de partir de Flaubert pour expliquer l'autonomie de la modernité littéraire, Lahire part de la situation de l'écrivain contemporain pour remonter le fil de l'hétéronomie de la modernité littéraire. À la figure exceptionnelle de Flaubert, Lahire substitue des figures 
autrement plus représentatives de cette hétéronomie de la littérature, comme celle du médecin-poète allemand Gottfried Benn et surtout celle de Franz Kafka, qui a constamment mené une double vie, tantôt comme écrivain, tantôt comme employé d'une compagnie d'assurance, l'expérience acquise dans ce domaine ayant bien sûr un effet direct sur sa pratique d'écrivain (et peut-être aussi vice versa). Pour Lahire, une écrasante majorité d'écrivains, quel que soit leur prestige, ont un second métier qui les fait vivre, qui leur permet d'écrire et qui, surtout, conditionne leur écriture au sens où celle-ci se nourrit de l'expérience acquise à travers l'exercice de cette autre profession. Même Flaubert, rappelle Lahire, insistait sur la nécessité pour l'écrivain de se frotter à tous les savoirs, de sortir du monde littéraire. La littérature n'a jamais été franchement un domaine clos, y compris donc au temps de Flaubert alors que l'idée d'autonomie se cristallise de la façon la plus évidente.

Le discours des écrivains contemporains donne une idée de la condition hétéronome de la littérature. On peut penser que la posture de l'écrivain-théoricien, de Baudelaire à Breton en passant par Zola et Mallarmé, traduit une valorisation très forte de l'autonomie de la littérature. De la même façon, on peut penser que l'effacement progressif de cette figure ou, si l'on veut dire cela de façon plus positive, que la montée en légitimité de l'écrivain-praticien, de l'écrivain qui commente sa pratique, correspond plutôt à une valorisation de l'hétéronomie de la littérature. L'écrivain contemporain n'a plus à démontrer que la littérature est un monde en soi, il n'a plus à faire de la «rupture» une sorte de cri de guerre pour marquer son indépendance à l'égard des pouvoirs politiques ou religieux : c'est là un combat déjà ancien en quelque sorte, un combat qui 
resurgit il est vrai de temps en temps, mais sous des formes résiduelles qui ne modifient guère le fait que rien de tout cela ne peut plus être qualifié de nouveau. La nouveauté n'est plus du côté de la rupture scandaleuse, et la vraie rupture n'est plus du côté de la revendication de la nouveauté. Le combat s'est donc déplacé sans qu'on en prenne vraiment acte, n'étant plus mené de manière spectaculaire par des écrivains producteurs de manifestes et de textes programmatiques destinés à faire école. Il est mené de façon plus diffuse, plus discrète sans doute, mais non moins profonde et réelle. Ce déplacement peut se décrire ainsi: l'autonomie de la littérature s'est tellement banalisée qu'elle a perdu sa force dramatique, de sorte que l'écrivain contemporain est plutôt porté à revendiquer la nécessité de sortir de la littérature, ou du moins de ne pas se laisser enfermer dans un discours autoréflexif qui n'aurait qu'une portée formelle ou esthétique. Il ne s'agit pas tant de renier l'autonomie que de partir d'elle pour aller vers autre chose. L'écrivain contemporain ne fait plus de la littérature un absolu, une fin en soi : il assume une certaine intransitivité propre à la littérature, mais au nom d'une vision plus large qui refuse de se définir en termes strictement esthétiques.

On peut penser que cela tient en partie au fait que l'écrivain ne se perçoit plus comme un « homme de lettres », un peu comme Réjean Ducharme lorsqu'il écrit en exergue à son roman Le Nez qui voque: "Je ne suis pas un homme de lettres: je suis un homme» $(1967$, p. 8). Une formule comme celle-ci résume la situation de l'écrivain québécois de façon saisissante, un écrivain qui n’a jamais cru à la valeur «autonomie de la littérature ». On peut lui donner une extension beaucoup plus grande à présent, bien au-delà du cas québécois qui, loin de Paris et des équilibres qui s'y maintiennent, exacerbe peut-être 
certaines des contradictions propres à l'ensemble du système : c'est l'écrivain en général qui ne veut pas être un homme de lettres. Une telle revendication, à rebours de la revendication d'autonomie formulée par un Flaubert, s'explique sur un plan sociologique par la situation générale qui prévaut: l'écrivain contemporain type a un second métier, il participe au monde autrement qu'à titre d'écrivain et il écrit depuis cette hétéronomie même.

Pour Lahire, il faut insister sur ce point crucial: la littérature n'a jamais été une sphère autonome structurée à la façon des autres sphères d'activité comme l'enseignement ou le journalisme. Ce qui caractérise la littérature, au contraire, c'est sa résistance au processus de professionnalisation qui détermine la plupart des autres sphères d'activité sociale et qui est à la base même de leur autonomie. L'univers littéraire est globalement «très peu professionnalisé et très faiblement rémunérateur » (2006, p. 12); il réunit des individus qui, en nette majorité, appartiennent à d'autres univers professionnels pour gagner leur vie. Pour faire comprendre la différence entre le domaine littéraire et, par exemple, le domaine de l'enseignement, Lahire rappelle qu'un professeur qui obtient une promotion, à moins d'une faute grave, ne va pas ensuite craindre d'être déclassé ou de perdre son statut social : il va progresser selon les normes fixées au sein de sa profession. Un universitaire ne peut pas revenir en arrière après être passé d'un échelon à un autre. En revanche, un écrivain (ou un artiste en général) peut très bien déchoir et tomber dans l'oubli après un départ fracassant. Le statut d'écrivain est incertain et toujours révocable. Aucun concours, aucun diplôme ne vient réguler l'accès à des postes d'écrivain (et l'absurdité même de la notion de poste d'écrivain montre bien l'inadéquation du 
vocabulaire bureaucratique pour parler du cas de l'écrivain). Il n'y a pas de normes professionnelles en littérature et un météorite sorti de nulle part peut obtenir plus de reconnaissance qu'un écrivain expérimenté. Ce qu'on a appelé la «professionnalisation de l'activité littéraire » ne ressemble donc que de très loin à ce qui se passe dans les institutions autrement plus structurées, comme l'est l'institution scolaire.

Cela ne veut pas dire que la littérature ne s'est pas malgré tout «autonomisée » : tout dépend du sens que l'on donne à la notion d' " autonomie ». Il est clair que les pratiques spécifiques à la littérature, que ce soit les jurys littéraires, les revues littéraires, les bourses réservées aux écrivains, etc., existent bel et bien et même de plus en plus depuis l'époque de Flaubert. On pourrait penser que cette prolifération de pratiques proprement littéraires renforce l'autonomie de la littérature et que le « champ littéraire », au sens de Bourdieu, n’a jamais été plus autonome qu'aujourd'hui. Mais ce serait une erreur, selon Lahire, pour qui la confusion vient de ce qu'on superpose deux sens distincts du mot "autonomie», selon qu'on désigne l'ensemble de pratiques spécifiques ou l'autonomie idéologique de la littérature à l'égard de tout ce qui est extra-littéraire. Selon lui, s'il est incontestable que les pratiques spécifiques existent dans le domaine littéraire, l'autonomie idéologique, elle, n'a jamais vraiment existé, la littérature passant de la tutelle religieuse ou politique à celle des lois du marché. Une telle distinction fait réfléchir et permet d'aboutir au paradoxe suivant: c'est au moment où les pratiques littéraires se spécialisent plus que jamais que l'autonomie de la littérature est dévalorisée aussi bien de l'intérieur (par les écrivains eux-mêmes) que de l'extérieur (par le discours social en général). 
L'un des corollaires de cela, c'est que la distinction bourdieusienne entre sphère de production restreinte et sphère de grande production ne semble plus opératoire pour décrire la situation de l'écrivain contemporain. Ce que les théoriciens de l'École de Francfort dénonçaient comme étant de la Kulturindustrie est devenu aujourd'hui une réalité courante qui ne scandalise plus personne: la littérature fonctionne bel et bien comme une industrie culturelle, et même les écrivains les plus prestigieux n'y échappent pas. La frontière symbolique séparant la sphère de grande production et la sphère de production restreinte n'est pas disparue, loin de là, mais elle n'a plus le caractère figé et définitif qu'elle avait au temps de Flaubert. Bernard Lahire écrit à ce sujet :

Les nouvelles conditions d'exercice du métier intellectuel ne reposent plus sur la "claire démarcation des sphères d'activité », mais sur une alternance entre des moments de "participation» et de "détachement» au sein du contexte familial, entre une présence intime et les « absences » mentales de celui qui étudie. (2006, p. 505)

De la même manière, le sociologue anglais Scott Lash constate que le postmodernisme se caractérise par ce qu'il appelle la «dédifférenciation ${ }^{1}{ }^{\prime}$ culturelle : les frontières entre culture d'élite et culture populaire se brouillent et on voit naître un public de masse pour la culture d'élite. Un écrivain-philosophe-critique comme Umberto Eco est aussi à l'aise pour parler de James Bond que des écrits de Borges. Le domaine littéraire s'est «démocratisé » ou, à tout le moins, il n'est plus «auratique» au sens que donnait à ce mot Walter Benjamin. Le poète n'est plus hanté par le désir de se préserver de «l'universel reportage » ou de donner « un sens plus

1 «If cultural modernization was a process of differentiation, then postmodernization is one of de-dedifferentiation. » (1990, p. 11) 
pur aux mots de la tribu » (Mallarmé). L'écrivain se sait immergé, qu'il le veuille ou non, dans le discours. De la même façon, ce n'est sans doute pas un hasard si la théorie du discours social défendue par Marc Angenot est apparue à l'époque contemporaine, cette théorie postulant que tout énoncé, du poème le plus hermétique au discours du politicien, fait partie d'un seul et même univers de discours.

Mais cette remise en question de l'autonomie de la littérature entraîne aussi d'autres conséquences, plus difficiles à mesurer. De façon générale, on peut dire que l'écrivain contemporain a parfaitement conscience à la fois des arabesques futiles de l'art pour l'art et du terrorisme de l'embrigadement qui s'est manifesté à divers moments de l'histoire littéraire, jusqu'à mai 68 , qui est la dernière révolution en date, celle dont nous sommes aujourd'hui les descendants plus ou moins directs. L'écrivain contemporain, hanté par cette double impasse, ne croit pas plus à ceci qu'à cela : l'autonomie littéraire n'est plus une conquête et n'a plus guère de sens en elle-même, mais le combat politique n'a pas beaucoup plus de sens s'il s'agit d'instrumentaliser la littérature. Conscient et même «hyperconscient » des apories de la littérature moderne tant il connaît la musique, tant il a lu Bourdieu pourrait-on dire en forme de boutade, cet écrivain ne cesse de jouer de sa lucidité même, et l'autonomie littéraire dont il hérite, il s'en réclame et s'en méfie tout à la fois.

\section{L'œuvre « partageable » de Pierre Michon}

Pour mettre un peu de chair autour de cette distinction quelque peu abstraite, évoquons l'exemple d'un écrivain contemporain 
français qui parle abondamment de sa pratique et qui cherche justement à penser la littérature, à penser sa propre pratique dans la lignée de Flaubert justement. Il s'agit de Pierre Michon, dont la critique a beaucoup parlé pour illustrer ce qu'il advient de la littérature française à l'ère contemporaine. Michon paraît résumer mieux que quiconque la posture paradoxale qui s'esquisse ici, notamment dans ses «propos sur la littérature » réunis récemment sous le titre Le Roi vient quand il veut. Le roi, c'est bien sûr la littérature elle-même (le « génie ${ }^{2}$ »), qui semble résumer à lui seul tout un monde de valeurs anciennes comme le roi - liées à la question du sens et du vrai. Peu importe si on l'appelle ou non: on ne sait jamais si le roi va venir ou non ; comme lui, la littérature — la vraie littérature vient quand elle veut.

Pierre Michon se réclame de l'avant-garde, celle qui a magnifié la posture de l'écrivain-théoricien, du Nouveau Roman jusqu'à Tel Quel. Il ne le fait pas toutefois de façon suiviste ou nostalgique, mais simplement en souhaitant tenir le pas gagné, en refusant l'idée de retour qui hante le discours métalittéraire. Il écrit notamment ceci : " Le prétendu retour au récit sert peutêtre de couverture à quelque chose de plus grave : on assiste au retour en force de la non-littérature sur le terrain même $d u$ littéraire; d'une facilité agressive qui n'avait plus guère de sol théorique sous les pieds depuis vingt ans.» (2007, p.15-16) Qu'entend-il par la «non-littérature »? C'est «l'increvable réaction poujadiste et hussarde contre les clercs », c'est-à-dire une sorte de populisme, de consensus populaire avec des postures publicitaires qui transforment l'écrivain en un

\footnotetext{
2 Le mot "génie " se retrouve ailleurs sous la plume de Michon, à côté de « snobisme » (Michon, 1997, p. 31).
} 
pourvoyeur de créativité au service des classes moyennes. Michon s'ennuie des Rimbaud, Lautréamont, Céline, Artaud et autres Thomas Bernhard. «Plus personne n'a les reins assez solides (ni le goût d'un risible martyre) pour occuper cette place-là. » (p. 17) Michon ne cesse de faire signe vers un certain héritage de la modernité radicale, qui comprend aussi des auteurs comme Proust ou Genet et surtout Faulkner, son père en littérature. Il s'ennuie d'écrivains qui incarnent au fond la grandeur souveraine de la littérature, sa capacité de résister à la «non-littérature », de marquer son territoire et de s'y tenir à distance des postures publicitaires qu'il dénonce.

À la lumière de cela, on pourrait penser que l'exemple de Michon contredit l'idée d'une «hétéronomisation» de la littérature, puisqu'il semble porter très haut le flambeau de la littérature au sens flaubertien. Or, c'est justement parce qu'il est le plus flaubertien des écrivains contemporains qu'il paraît révélateur du déplacement qui s'opère, dans la mesure où même lui, même cet admirateur de Flaubert, va rejeter de façon explicite les mignardises de l'art pour l'art et le culte de la nouveauté. Ce rejet prend d'autant plus de sens qu'il s'exprime sur fond d'adhésion. Quand Michon parle des écrivains les plus ouvertement modernes, il reconnaît d'abord une immense dette. Voici comment il décrit par exemple l'avant-garde :

J'ai longtemps été fasciné par l'avant-garde [...] et je crois que je lui dois beaucoup. Elle nous disait que la littérature est perpétuelle rupture; que son objet est impossible; que le bond hors du consensus en est une condition essentielle. Elle entretient la colère, la nécessité offensive, sans laquelle toute écriture me paraît nulle et non avenue; et, comme elle était en guerre accessoirement contre la République des Lettres mais aussi contre le monde, elle réaffirmait sans cesse les liens de l'esthétique et de l'éthique. Elle rejetait au néant tout ce que 
nous détestions : elle était la mauvaise conscience du monde comme il va et de la littérature de loisir qu'elle délégitimait ; et celle-ci survivait sans alibi, plus ou moins honteuse. (2007, p. 13-14)

L'avant-garde devient ainsi, pour lui, une sorte de "surmoi littéraire » (p. 14), comme si elle lui rappelait sa dette envers la littérature, envers l'opacité de la littérature. Michon écrit plus loin: "Je crois que les plus grandes œuvres sont opaques et ajoutent à l'opacité du monde»(p.60). Dans un deuxième temps, il met cet héritage à distance. Lorsqu'il parle de Rimbaud, il insiste sur le fait que les écrivains modernes sont tous des fils sans père. Il ne veut rien savoir d'un art qui romprait pour rompre, qui tomberait dans l'art pour l'art. Rimbaud est admirable parce qu'il a, le premier, cassé la baraque de la modernité, parce qu'il s'est moqué des modernes devenus les «néo-académiques en puissance» (p.55), les ampoulés de la modernité, les amateurs de nouveauté. Pour Michon, l'œuvre doit être « partageable » (p. 56). Il écrit : « Mon projet, s'il existe, n'est pas d'ordre esthétique » (p. 56). Non pas rompre avec le monde (ce qui est désormais jugé risible et " suicidaire »), mais " donner à partager un amour possible du monde » (p. 56), formule on ne peut moins rimbaldienne. Sans renier la révolution du langage poétique (le "pas gagné »), Michon parle de conciliation avec le monde, comme si la littérature devait à présent combler une distance devenue insensée et préciser son combat. Ce que Michon rejette, ce n'est plus le monde bourgeois, mais le poujadisme, la démagogie, le goût pour la facilité, les habitudes publicitaires, la mollesse consensuelle.

Dans un des entretiens du volume, publié initialement dans Le Magazine littéraire en 1997, l'intervieweur Thierry 
Bayle demande à Michon s'il n'y a pas chez lui une "tentation du silence », une fascination pour un "monde sans écriture », comme si l'écrivain entendait au fond de lui une voix cynique du type : «Cause toujours ». Il n'en faut pas beaucoup pour que Michon se sente pris du syndrome de l'imposteur, un syndrome qu'il associe à la condition même de l'écrivain moderne, puisque ce dernier ne tire sa légitimité que de lui-même et recherche partout des signes pour valider sa fragile ambition. Tout se passe comme si Michon avait intériorisé le point de vue de Bourdieu, comme quoi le commentaire de l'écrivain contemporain sur sa pratique est désormais médiatisé, instruit par la connaissance qu'il a des travaux sociologiques qui ont cherché à l'expliquer :

Sans doute qu'à partir du moment où la littérature s'est constituée comme fin en soi, sans Dieu, sans justification extérieure, sans idéologie qui la soutienne, en champ autonome comme dit Bourdieu, c'est-à-dire en gros avec Flaubert et Mallarmé, ou un peu avant, à partir de ce moment tout écrivain a été un imposteur, puisqu'il ne pouvait s'autoriser que de luimême. Mais c'est aussi ce qui fait la force de la littérature depuis ces gens-là : tout écrivain se tient seul face à la totalité de l'être, sans béquilles. (2007, p. 146-147)

Autrement dit : c'est parce que l'écrivain moderne se méfie des systèmes extérieurs et abstraits de nature religieuse ou politique qu'il place sa confiance dans la littérature elle-même, en tant que «fin en soi ». La littérature n'est plus instrumentalisée, mais cette autonomie typique de la modernité a un coût et ce coût, c'est précisément ce sentiment avoué d'imposture. Tout écrivain moderne éprouve ce sentiment, nous dit Michon, mais on peut penser que la lucidité même dont il fait preuve, en objectivant ainsi sa propre situation à la façon d'un sociologue, témoigne d'une capacité réflexive accrue, 
perçue non plus comme une forme d'indépendance conquise, mais comme un fragile équilibre de l'être, désormais condamné à se tenir « sans béquilles ».

La nouveauté de la condition littéraire propre à l'écrivain contemporain, pour être saisie de façon globale, devrait être rapportée à des transformations qui affectent la façon même dont l'individu se perçoit au sein de son milieu ou de sa société, non seulement dans l'espace littéraire, mais également dans les autres sphères d'activité sociale. Il est peut-être utile de la rapprocher de ce que le sociologue anglais Anthony Giddens décrit lorsqu'il parle de la "réflexivité extrême » qu'exigent les « relations pures » qui caractérisent, selon lui, l'individu dans la "modernité tardive», c'est-à-dire à partir de la deuxième moitié du XXe siècle («the extreme reflexivity of late modernity », 1991, p. 29). Pour Giddens, les systèmes politiques et institutionnels mis en place pour compenser la perte de foyers de sécurité ontologique (les relations de parenté, l'appartenance à des communautés locales, la croyance dans des cosmologies religieuses et l'autorité de la tradition) demeurent abstraits, désincarnés et lointains. L'individu compense alors l'affaiblissement de la sécurité ontologique en surinvestissant le domaine affectif, en plaçant sa confiance dans le développement de relations interpersonnelles d'amitié et d'amour qui constituent une réponse à la dérive impersonnelle des échanges sociaux. Ces « relations pures » se veulent à l'abri de conditionnement externe et trouvent leur valeur en ellesmêmes, puisqu'elles ne dépendent pas d'obligations, mais seulement du bien-être émotionnel qu'elles procurent à l'individu. Cette pureté a un prix toutefois, tout comme l'autonomie de la littérature dont parlait Michon, qui est la fragilité même de la relation dont la valeur doit sans cesse être 
réaffirmée par chaque individu, libre de maintenir ou non le lien qui le définit. La liberté maximale entraîne une précarité du lien social, comme c'est le cas aussi chez l'écrivain, qui éprouve le besoin de marquer la fonction de la littérature dès lors qu'il n'y a plus de justification extérieure et que l'écrivain se trouve malgré lui "sans béquilles». Quand Michon exige de l'œuvre qu'elle soit "partageable», quand il souhaite "donner à partager un amour possible du monde », il ne tombe pas dans un romantisme anachronique et naï, mais prend acte du fait que, si la littérature se constitue depuis déjà longtemps comme une "fin en soi», la véritable autonomie ne saurait se payer qu'au prix fort, par la mauvaise conscience qui s'empare alors de l'écrivain toujours menacé de paraître futile et coupé du monde aux yeux d'autrui comme à ses propres yeux, comme un imposteur, c'est-à-dire comme quelqu'un qui s'attribue une valeur sociale dont il ne parvient pas à ne pas douter par ailleurs.

Ainsi, la question de l'autonomie ne se pose plus de la même façon à l'écrivain contemporain. Au temps de Flaubert, il s'agissait de revendiquer une liberté nouvelle, qui permettait d'écrire un « livre sur rien » et d'opposer aux règles morales des principes esthétiques. Au temps de Michon, c'est-à-dire à notre époque, il s'agit d'assumer la logique de l'autoréflexivité héritée de Flaubert et des autres écrivains modernistes mais en la transportant nettement du côté du récit de soi, en conciliant l'expérience de l'écriture (y compris le « surmoi littéraire » que représente l'avant-garde) et l'expérience intime (non littéraire) du monde. L'autonomie n'est plus une conquête, mais un héritage qui n'a de sens que si on lui oppose un désir nouveau, 
qui transforme l'imposture moderne en quelque chose de positif, mais qui ne soit sanctionnable par aucune autorité. « Le roi vient quand il veut » signifie que la littérature est autonome, en ce sens que le roi n'est contraint par aucune autorité extérieure; cela signifie aussi, à l'inverse, que l'écrivain est tenu de faire sans cesse son autocritique, comme le fait Michon dans ses entretiens lorsqu'il admet se sentir imposteur. Cette autocritique inclut le refus d'une certaine littérature complaisante et la revendication d'une nécessaire hétéronomie dans la mesure où l'écrivain appelle à autre chose qu'à la littérature (un «amour possible du monde») pour mieux se délivrer de ce qu'il appelle la non-littérature, qui a désormais envahi son propre territoire. À la logique de la distinction qui sert de matrice explicative aux stratégies d'auteur dans la théorie du champ littéraire se substitue ainsi une logique d'autoréflexivité par laquelle l'écrivain s'accuse d'être un imposteur à force de vouloir se présenter comme écrivain. Sans inférer qu'il s'agit d'un principe général, on peut penser qu'un tel souci de «dé-différenciation», de «dé-distinction » correspond bien à la situation réelle des écrivains contemporains telle qu'analysée par Bernard Lahire et n'est pas sans effet sur leur façon d'écrire. Une telle hétéronomie de la littérature pourrait se résumer par une formule qui se trouve dans un roman récent d'Emmanuel Carrère, D'autres vies que la mienne, qui met en scène l'écrivain lui-même arrivant au bout de son aventure et concluant : «Ah, et puis : je préfère ce qui me rapproche des autres hommes à ce qui m'en distingue. Cela aussi est nouveau. » (2009, p. 308) 


\section{Bibliographie}

ANGENOT, Marc (1989). 1889. Un état du discours social, Longueuil, Le Préambule.

BENJAMin, Walter (2000 [1935]). «L'œuvre d'art à l'époque de sa reproductibilité », dans OEuvres III, Paris, Gallimard.

BouRdiEu, Pierre (1992). Les Règles de l'art. Genèse et structure du champ littéraire, Paris, Seuil.

CARRÈRE, Emmanuel (2009). D'autres vies que la mienne, Paris, P.O.L.

DuCHARME, Réjean (1967). Le Nez qui voque, Paris, Gallimard.

GidDENS, Anthony (1991). Modernity and Self-Identity. Self and Society in the Late Modern Age, Stanford, Stanford University Press.

LAHIRE, Bernard (2006). La Condition littéraire. La double vie des écrivains, Paris, La Découverte, coll. «Textes à l'appui. Série Laboratoire des sciences sociales ».

LASH, Scott (1990). Sociology of Postmodernism, London and New York, Routledge.

Michon, Pierre (1997). Trois auteurs, Lagrasse, Verdier.

- (2007). Le Roi vient quand il veut. Propos sur la littérature, textes réunis et édités par Agnès Castiglione avec la participation de Pierre-Marc de Biasi, Paris, Albin Michel. 


\title{
Résumé
}

Ce texte examine d'abord la question de l'hétéronomie de la littérature à partir d'une enquête menée par le sociologue Bernard Lahire (La Condition littéraire. Ladouble vie des écrivains). Celui-ci montre que l'écrivain contemporain assume une certaine intransitivité propre à la littérature, mais au nom d'une vision plus large qui va au-delà de l'autonomie de la littérature. Ce texte étudie ensuite le cas de Pierre Michon se réclamant de figures avant-gardistes capables de résister à la « non-littérature ». Tout en polémiquant avec le discours actuel, Michon fait sienne toutefois une préoccupation éthique propre à la littérature contemporaine. Pour lui, l'œuvre doit être « partageable» et appelle à un "amour possible du monde», rejoignant par là l'idée d'hétéronomie de la littérature.

\begin{abstract}
The first part of this study raises the question of the frontier between literature and non-literature based on a recent research conducted by sociologist Bernard Lahire (La Condition littéraire. Ladouble vie des écrivains). While defending the autonomy of literature, contemporary writers, as Lahire demonstrates, share a vision that goes way beyond aesthetic questions. The second part of this study illustrates this vision with the example of Pierre Michon's "Propos sur la littérature" (Le roi vient quand il veut). Michon insists on the avant-garde's heritage and opposes the invasion of publicity and nonliterature inside literature's domain. For him, the main goal of work of art is to be shared with others and to embrace "un amour possible du monde".
\end{abstract}

